\title{
Mobilizing animal models against a pandemic
}

\author{
As COVID-19 continues to spread, the urgency of the hunt for effective therapeutics and vaccines has likewise \\ grown. As with past outbreaks, animal models will be an important component of efforts to understand how the \\ SARS-CoV-2 virus causes disease, and how best to defeat it.
}

\section{Michael Eisenstein}

T here are no guarantees that researchers will be able to find a ready animal surrogate to model human disease. For example, Paul McCray, who studies pulmonary diseases at the University of Iowa, notes that researchers still lack good models for well-known menaces such as respiratory syncytial virus (RSV). This pathogen, which was first discovered in the 1950s, typically causes mild cold-like symptoms. But severe infection can be dangerous, especially to children-recent estimates suggest that RSV is responsible for 33 million hospitalizations and 60,000 deaths amongst young children annually ${ }^{1}$. "But if you put RSV in a mouse, you just get some mild disease," he says. "In some other animals, it doesn't even complete its replication cycle." Even in a best-case scenario, it is highly unlikely that any one animal model will capture the full spectrum of disease pathology seen in humans.

Whenever a new viral threat emerges, researchers often find themselves back at square one in terms of identifying which animal species might be best suited to the task of replicating human pathology. This can be a trial and error process-and in some cases, there may be no good solutions. On the other hand, the researchers mobilized against COVID-19 have an advantage: the knowledge gained from recent skirmishes with other coronaviruses. These include the 2012 outbreak of Middle Eastern respiratory syndrome (MERS) and the 2003 outbreak of severe acute respiratory syndrome (SARS), whose causative pathogen, SARS-CoV, is a close genetic relative of the current threat.

"An emerging virus that is similar to other known viruses can make things easier," says Barry Rockx, a virologist at Erasmus Medical College in the Netherlands. But considerable experimentation will still be necessary to home in on the most effective proving ground for this front of the war against COVID-19.

\section{Making the most of mice}

The mouse is usually the first place scientists turn. These animals are readily available,

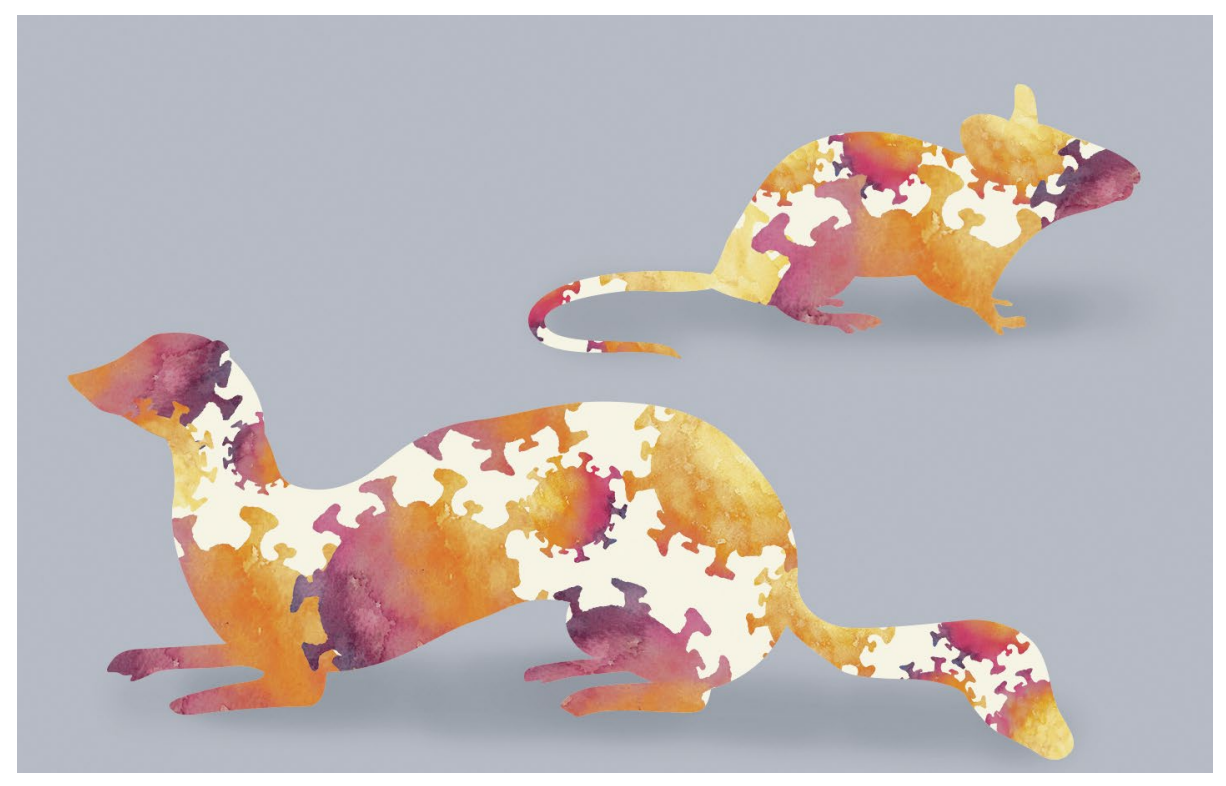

Credit: Marina Spence \& Erin Dewalt / Springer Nature

easy to maintain and study, and extremely well characterized, even if they don't succumb to many of the pathogens that sicken humans. "In a lot of cases, wild-type mice may be susceptible in the sense that the virus can replicate, but very often it will not cause disease," explains Rockx.

Mice and humans share most of the same genes and produce highly similar proteinson average, our protein-coding sequences are $85 \%$ identical. But even if mice express the same proteins that viruses use to bind and infiltrate human cells, those murine versions may differ sufficiently in their amino acid sequence to prevent efficient infection. This was the case for SARS-CoV, which binds strongly to the human version of a protein called angiotensin-converting enzyme 2 (ACE2) but has poor affinity for the mouse homologue. As a solution, several groups generated genetically modified mice that express the human form of ACE2. McCray and his University of Iowa colleague Stanley Perlman were among those who achieved some success with this approach ${ }^{2}$.
"We could get virus replication in the lungs and some inflammatory disease, but it wasn't exactly a copy of the human disease," says McCray. "In many cases, the animals also developed severe encephalitis, which was fatal." Human SARS patients, however, were succumbing to pneumonia, not brain inflammation.

MERS-CoV proved even trickier. This coronavirus targets a protein known as dipeptidyl peptidase 4 (DPP4), and mouse DPP4 differs so substantially from the human version as to provide no good purchase for the virus. The human protein also proved toxic in mouse, as Chien-Te Tseng and colleagues at the University of Texas Medical Branch found while trying to develop a transgenic model. "If you overexpress human DPP4 in transgenic mice, they could give birth, but they were dying prematurely... probably within a few days after birth," says Tseng. As a consequence, they could only work with transgenic mice that weakly expressed this protein, although these animals did grow 


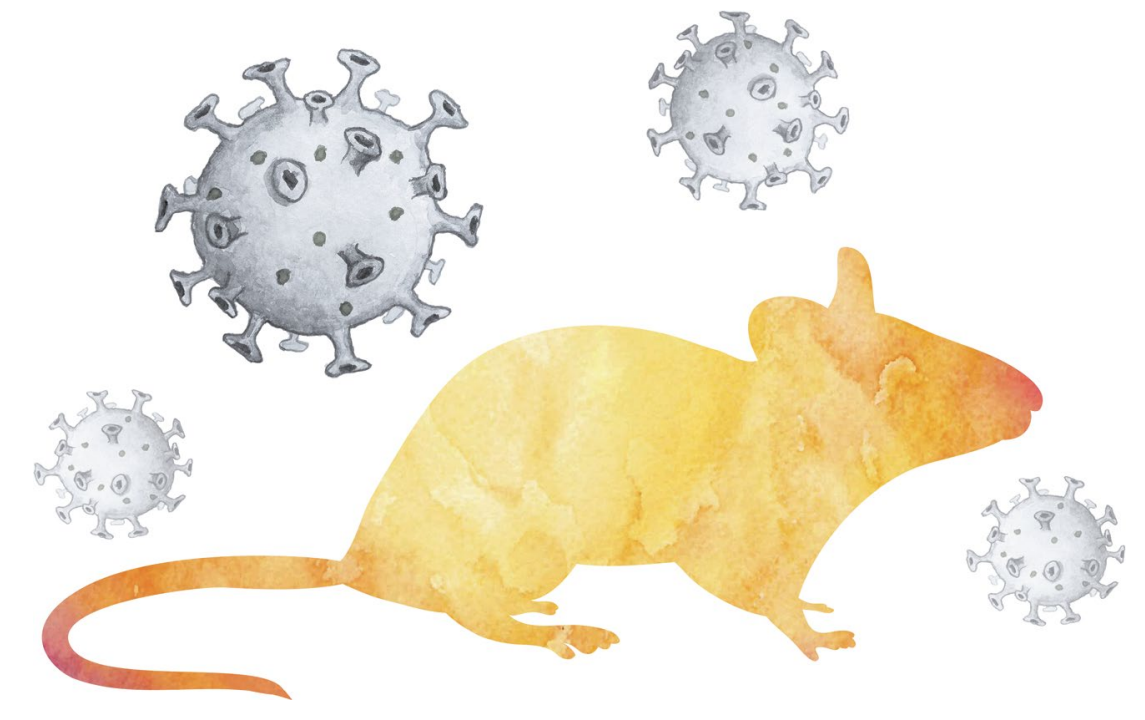

Credit: Marina Spence \& Erin Dewalt / Springer Nature

sick and replicate some features of human MERS disease, including progressive pneumonia ${ }^{3}$.

McCray and Perlman took a different tack rooted in McCray's prior experience with gene therapy, using an adenovirus vector to selectively induce targeted expression of human DPP4 exclusively in the respiratory tracts of mice". "That allowed us a shortcut for sensitizing mice to the virus," says McCray. These animals were readily infected by MERS-CoV, and developed manifestations of pneumonia similar to those seen in human patients.

As an alternative to genetically modifying animals, one can 'train' viruses to recognize a new host via a process called serial passaging. This entails repeated cycles of infecting mice, and then using viral isolates from those animals to infect a new set of mice. The goal is to rapidly evolve the virus over the course of several weeks, selecting for those viruses that take the strongest hold in their new animal host. Ralph Baric's team at the University of North Carolina used this approach to successfully model SARS disease in mice. "It took about 15 to 20 passages for the virus to become lethal in mice," says Rockx, who worked in the Baric lab as a postdoc. This approach can reveal valuable information about virus features that determine host specificity, but may not always deliver a faithful model of human disease. For example, Rockx notes that passaging yielded mixed results in mouse models of Ebola ${ }^{6}$. "This identified two or three mutations responsible for infection," he says. "But a lot of the disease seen in humans is not recapitulated in this mouse model."

\section{A partial picture}

In the context of coronaviruses, these models have given researchers a useful jumping-off point for characterizing how key steps of infection unfold, and for early-stage investigation of clinical interventions. "Our transgenic mice clearly indicate that type I and type II pneumocytes-these are the epithelial cells lining the alveoli-are the main targets supporting viral replication in the lung," says Tseng. Such infections can also elicit a human-like immune response to both SARS- and MERS-CoV, including the severe inflammatory injury that can occur in the lung as collateral damage from this counterattack.

One of the hallmarks of both SARS and COVID-19 is the apparently increased risk of severe or fatal disease in elderly individuals. Researchers led by Kanta Subbarao-now at the Peter Doherty Institute for Infection and Immunity in Melbourne, Australia-likewise demonstrated in $2005^{7}$ that they could better replicate the impact of human SARS by working with year-old mice rather than young animals. Rockx observed the same effect in his work in the Baric lab". "The immune response in young mice seems to be more controlled," he says. "With aged mice, that response doesn't seem to be as controlled... and you can get what people call a 'cytokine storm."' This is a valuable insight, given that such an uncontrolled immune response has been linked to deaths in human coronavirus infection.

Even models with mild disease symptoms can be informative, revealing tissues and cell types where the virus prefers to bind and infiltrate, or serving as a low-pass filter' for drug or vaccine testing. "If there are treatments that actually enhance the disease, you will not see that necessarily in a very virulent model where the animals are already dying," says Rockx. He notes that this has been observed in studies of dengue, where an antibody response elicited against the virus can actually promote future infection rather than preventing it. Conversely, Tseng notes that an outright failure to generate a meaningful antiviral response in mice should give researchers pause about scaling up to more expensive large animal models or clinical testing.

But one cannot rely too heavily on mice, either. As mentioned above, many mice that develop lung disease in successful models of MERS and SARS actually succumb to neurologic disease, which is not typical in actual patients. Tseng notes this is not exclusively a problem for coronaviruses, either. "We see this with lots of mouse models, such as for influenza," he says. "If you inoculate intranasally, quite often the viruses migrate through to the brain."

As has proven to be the case with countless other diseases, researchers should temper their optimism over signs of effective protection against infection in mice. Jean Patterson, a virologist at the Texas Biomedical Research Institute, points to the ongoing struggle to develop an effective herpes vaccine, even after multiple promising starts in mouse studies. "We've saved a lot of mice from herpes simplex virus-but that isn't our goal," she says.

\section{Bigger and better}

Some groups have turned to alternative small-animal models as a complement to mice. The Syrian golden hamster, for example, has proven to be an excellent model for SARS-CoV infection. Ferrets, with similar respiratory tract physiology to humans, are commonly used as a model for influenza, and a number of groups have found them a good match for more exotic emerging viral threats. For example, Rockx and his colleagues have been using ferrets to study Hendra and Nipah viruses, a pair of dangerous and incurable zoonotic pathogens that-fortunately-have only seen limited human spread to date.

But as our closest evolutionary relatives, nonhuman primates may offer the best solution for modeling diseases for which no other animal surrogate is sufficient. "You use nonhuman primate models only if it's absolutely necessary and it will give you the information you need to help in blocking the progression of human disease," says Patterson, who has worked with marmosets 


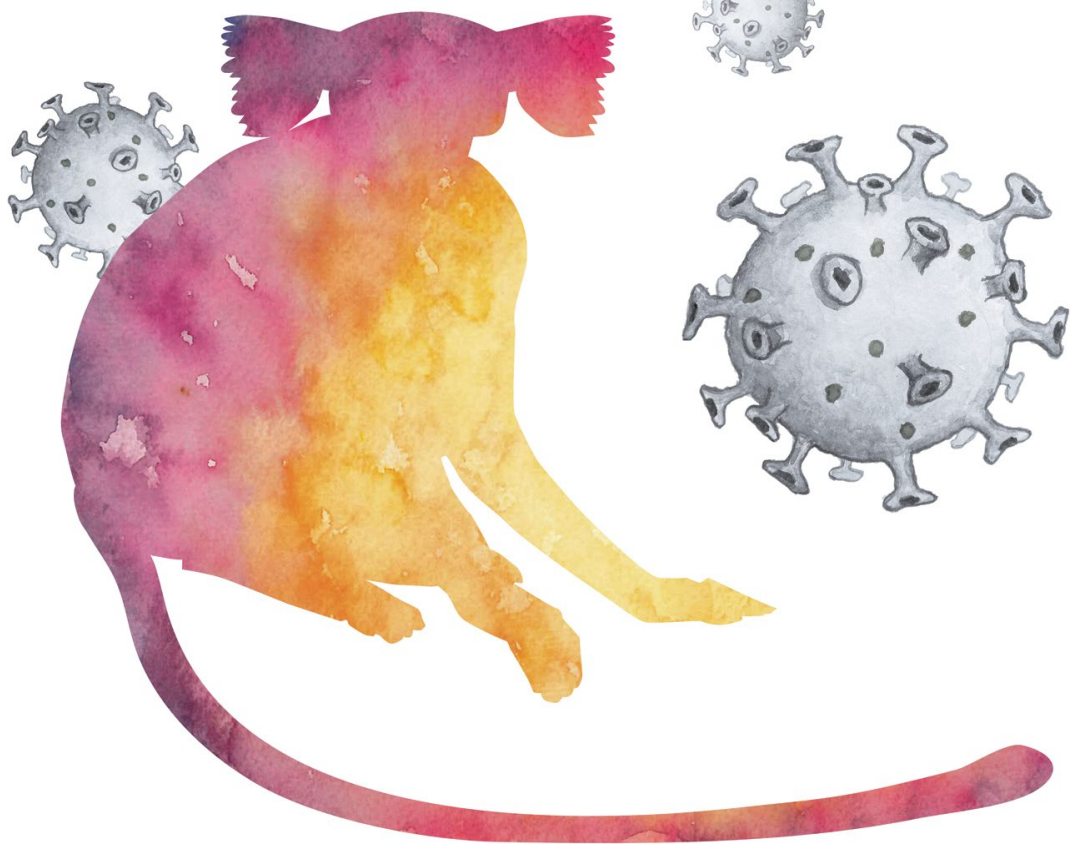

Credit: Marina Spence \& Erin Dewalt / Springer Nature

and macaques to study a variety of emerging viral threats in the past 20 years.

Patterson's team has had success in modeling the hemorrhagic diseases caused by the Ebola and Lassa viruses with marmosets, and most recently, in studying the risk to pregnant women from Zika. "Marmosets are interesting reproductivelythey have a baby, and then it is possible to get pregnant again in another two weeks, while other nonhuman primates only breed once a year," says Patterson. She and her collaborators demonstrated that pregnant marmosets exposed to Zika were prone to spontaneous miscarriage and abnormalities in fetal neurological development, and hypothesized that the placenta represents a major target for viral infection 9 . This model could thus give researchers a unique opportunity to study an outcome of Zika infection that has confounded and alarmed the medical community in a species whose prenatal development and gestation somewhat mirrors our own.

With coronaviruses, outcomes in nonhuman primates have proven a bit more unpredictable. On the one hand, several groups have replicated varying levels of respiratory disease from MERS-CoV in macaques and marmosets, and have even used these nonhuman primate models for preclinical testing of promising therapeutics ${ }^{10}$. But the picture for SARS-CoV is murkier. "SARS doesn't do much in marmosets," says Patterson, noting that initial indications of respiratory disease from one study could not consistently be replicated in subsequent efforts. And although SARS-CoV can infect cynomolgus macaques and trigger pathology in the lungs, it generally produces much milder disease than is typically seen in patients ${ }^{11}$.
Credit: Marina Spence \& Erin Dewalt / Springer Nature

\section{Forewarned is forearmed}

So where does this leave us in the battle against COVID-19? Fortunately, researchers around the world have mobilized quickly and collaboratively to counter the pandemic, and drawn on lessons learned from past experience. "With this outbreak, the genome sequence was known much faster than we could get an isolate to work with," says Rockx. "And just based on this sequence data and previous knowledge from SARS, people could quickly determine what the potential receptor was."

As luck would have it, the virus responsible for the current pandemic, SARS-CoV-2, attacks the same cellular receptor as SARS-CoV-the protein ACE2. McCray's lab is now drawing on the same toolbox they used to study MERS and SARS to tackle this new virus. Their first move was to use the same adenovirus gene therapy approach they employed with MERS, which enables the rapid generation of infectable models without time-consuming gene modification procedures. "We're already seeing that it works similar to what we saw with DPP4 in MERS," says McCray. In parallel, his group and others are exploiting insights about SARS-CoV-2's molecular interactions with ACE2 to generate mice with 'humanized' versions of this receptor, which might enable efficient infection. "It turns out you only have to introduce a few mutations into the mouse ACE2," says McCray, "and that can be done using CRISPR genome-editing technology."

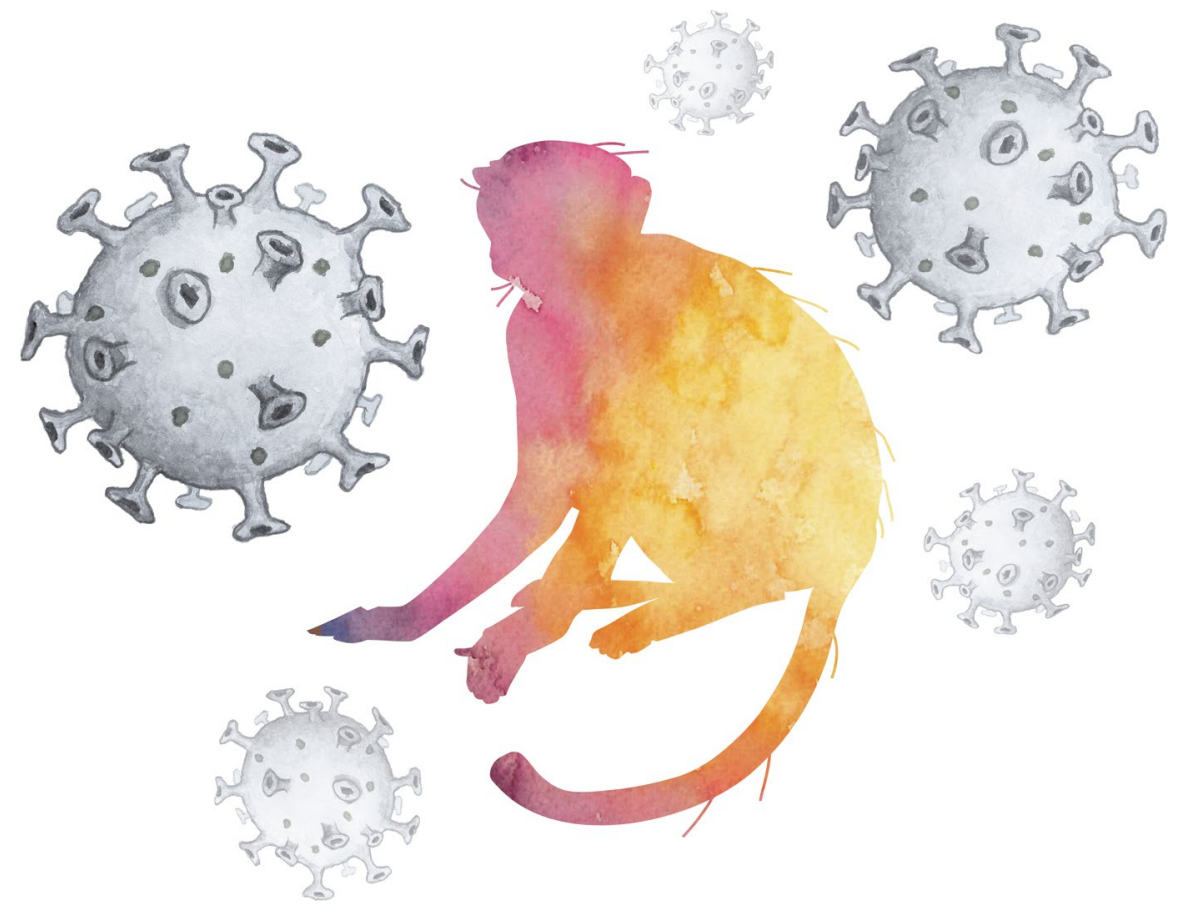


CRISPR is a molecular biology tool that allows researchers to rapidly introduce precisely targeted changes to genomic DNA. This greatly accelerates the development of genetically engineered mice relative to conventional approaches, which entail a more extensive process of genetic manipulation and breeding to produce transgenic animals.

Tseng is looking to model COVID-19 with human ACE2-expressing transgenic mice as well, but he has also partnered with collaborators at the Galveston National Laboratory in Texas to test a host of other model species with a track record of SARS-CoV susceptibility. These include both Syrian hamsters, shown to develop SARS-like disease, and ferrets, which generally exhibit mild symptoms from SARS-CoV but can still be infected and transmit the virus to other animals. "Our ferret challenge study is still ongoing for COVID-19... but the bottom line is, we do see some clinical disease," says Tseng, although he notes that high titers of infection appear to be needed to achieve this effect.

Given that cynomolgus macaques have proven susceptible to infection with both SARS-CoV and MERS-CoV, Rockx and colleagues recently ran a study to compare outcomes from intranasal
SARS-CoV-2 infection relative to these other coronaviruses ${ }^{12}$. "We found shedding of virus in the nasal and throat swabs, suggesting that there's at least some replication in the upper respiratory tract," he says. "We also found virus replication in the lower lungs, and in two animals, we saw some macroscopic lesions." More generally, he noted that SARS-CoV-2 tends to infect the same respiratory cell types and tissues as SARS-CoV, but appears to inflict less damage than that virus.

Patterson is generally discouraged by past data from coronavirus infection in marmosets and macaques, and has instead opted to try a more unconventional primate-baboons. She notes that there is some evidence to suggest that these nonhuman primates may be prone to some of the same respiratory infections as humans-including at least one instance where her group uncovered evidence of transmission of an airway-infecting adenovirus between baboons in one animal facility and the animal care staff. "It's a shot in the dark," she says. "People have tried SARS over and over in all kinds of models, but no one has tried baboons."

She doesn't expect this one new effort to be a game changer on its own. "I know most primate labs have just about given up on finding a good lethal model in nonhuman primates unless COVID turns out to be really different from SARS," says Patterson. But if it can replicate even a few key features of the disease relevant to treatment or prophylaxis, that would justify the gambleparticularly in an all-hands-on-deck situation like the current pandemic.

"I would anticipate that for COVID-19, there is no single animal model that will fully reflect the human disease," says Tseng. "But if we combine all these animal studies together... we can probably get a good picture of the pathogenesis."

\section{Michael Eisenstein ${ }^{凶}$}

Freelance science writer, Philadelphia, PA, USA.

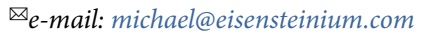

Published online: 12 May 2020 https://doi.org/10.1038/s41684-020-0558-5

\section{References}

1. Karron, R. A. \& Black, R. E. Lancet 390, 917-918 (2017).

2. McCray, P. B. et al. J. Virol 81, 813-821 (2007).

3. Agrawal, A. S. et al. J. Virol 89, 3659-3670 (2015).

4. Zhao, J. et al. Proc Natl Acad USA 111, 4970-4975 (2014).

5. Roberts, A. et al. PLoS Pathog 3, e5 (2007).

6. Chan, M. et al. Viruses 11, E987 (2019).

7. Roberts, A. et al. J. Virol 79, 5833-5838 (2005).

8. Rockx, B. et al. J. Virol 83, 7062-7074 (2009).

9. Seferovic, M. et al. Sci Rep 8, 6851 (2018).

10. Gretebeck, L. S. \& Subbarao, K. Curr Opin Virol 13, 123-129 (2015)

11. Rockx, B. et al. PLoS One 6, e18558 (2011).

12. Rockx, B. et al. Science 17, eabb7314 (2020) 